\title{
Nuclear Magnetic Resonance of a Layered Organic-Inorganic Hybrid System $\left(\mathrm{C}_{8} \mathrm{H}_{17} \mathrm{NH}_{3}\right)_{2} \mathrm{SnCl}_{6}$
}

\author{
Kyu Won Lee and Cheol Eui Lee* \\ Department of Physics, Korea University, Seoul 136-701, Korea
}

(Received 26 January 2004)

\begin{abstract}
Three successive phase transitions in bis- $n$-octhylammonium hexachlorostannate, $\left(n-\mathrm{C}_{8} \mathrm{H}_{17} \mathrm{NH}_{3}\right)_{2} \mathrm{SnCl}_{6}$, were studied by means of the ${ }^{1} \mathrm{H}$ nuclear magnetic resonance linewidth and spin-lattice relaxation measurements. Unlike the compounds with longer hydrocarbon chains, the order-disorder and conformational nature were found to coexist in the phase transitions.
\end{abstract}

Key words : layered organic-inorganic hybrid system, nuclear magnetic resonance, phase transitions

\section{Introduction}

The hexahalometallates of the general formula $\mathrm{A}_{2} \mathrm{MX}_{6}$ $\left(\mathrm{A}=\mathrm{K}^{+}, \mathrm{Rb}^{+}, \mathrm{Cs}^{+}, \mathrm{NH}_{4}^{+} ; \mathrm{M}=\mathrm{Pd}, \mathrm{Pt}, \mathrm{Sn}, \mathrm{Pb}, \mathrm{Re}, \mathrm{Se}, \mathrm{Te}\right.$, $\mathrm{Ir}, \mathrm{Os}, \cdots ; \mathrm{X}=\mathrm{Cl}, \mathrm{Br}, \mathrm{I})$ normally crystalize in the cubic antiflurite structure with space group $\mathrm{F} 3 m 3 m\left(\mathrm{O}_{h}{ }^{5}\right)$ in the high temperature phase $[1,2]$. They usually undergo structural phase transitions lowering their symmetry with decreasing temperature. If $\mathrm{A}$ is replaced by alkylammonium ion, $\left(\mathrm{R}_{n} \mathrm{NH}_{4-n}\right)_{2} \mathrm{MX}_{6}$ type compounds are formed and the dimensionality of the overall structure is reduced from $3 \mathrm{D}$ to 2D. Several examples have been reported for the $\mathrm{Sn}, \mathrm{Pt}$ and Te metals [3-6].

The bis- $n$-alkylammonium hexachlorostannates ( $n$ $\left.\mathrm{C}_{n} \mathrm{H}_{2 n+1} \mathrm{NH}_{3}\right)_{2} \mathrm{SnCl}_{6}\left(\mathrm{C}_{n} \mathrm{Sn}\right.$ for short $)$ are layer compounds, where the $\mathrm{SnCl}_{6}{ }^{2-}$ octahedra do not form a $2 \mathrm{D}$ macroanion but exist separately [2-4]. The $\mathrm{NH}_{3}$ group of the alkylammonium ion links the three closest octahedra through equivalent hydrogen bonds of the $\mathrm{N}-\mathrm{H} \cdots \mathrm{Cl}$ type, forming a layer. The distance between the ammonium groups or between the tin atoms in $\mathrm{C}_{n} \mathrm{Sn}$ is large (7.3 7.5 $\AA$, depending on the chain length) for interdigitated interlayer alkylchains. The alkylammonium groups are statically disordered around the three fold-axes at $(1 / 3,2 /$ $3, z)$ and $(2 / 3,1 / 3, \bar{z})$, with the alkylchains alternately pointing upwards and downwards [4].

In contrast to the case of bis- $n$-alkylammonium tetrashlorometallates $\left(n-\mathrm{C}_{n} \mathrm{H}_{2 n+1} \mathrm{NH}_{3}\right)_{2} \mathrm{MCl}_{4}$ with $\mathrm{M}=\mathrm{Cd}, \mathrm{Cu}$,

"Corresponding author: Fax: +82-2-3290-3098, e-mail: rscel@korea. ic. kr and $\mathrm{Mn}(\mathrm{CnM})$ [7-9], little information is available on the phase transition of $\mathrm{C}_{n} \mathrm{Sn}[2,4-6]$. In our previous ${ }^{1} \mathrm{H}$ nuclear magnetic resonance (NMR) studies of C10Sn and C18Sn, two phase transitions, i.e., order-disorder and conformational transitions, were clearly found and the molecular motions of the methyl and ammonium groups, and some defects were characterized in each phase [1012]. Although both CloSn and C18Sn show a very similar transition sequence, the transitional behaviors are quite different from each other. In the ${ }^{1} \mathrm{H}$ NMR study of C12Sn [13], the typical transition sequence were also observed as in Cl0Sn and C18Sn. The transitional behavior and the molecular motions in C12Sn were very similar to those in C10Sn. However, the transition sequence in $\mathrm{CnSn}$ with $n \leq 8$ are quite different from that in CnSn with $n \geq 10$ and the nature of the transitions are not known for $4 \leq n \leq 8[5,6]$. Nonetheless, it can be expected that the molecular motions in $\mathrm{C} 8 \mathrm{Sn}$ are similar to those in C10Sn, in spite of the distinct transition sequence. In this work, ${ }^{1} \mathrm{H}$ NMR was employed for the study of phase transitions and molecular motions in C8Sn.

\section{Experiment}

The C8Sn sample used in this work was synthesized with much care to avoid impurities by the chemical reaction: $2\left(n-\mathrm{C}_{8} \mathrm{H}_{17} \mathrm{NH}_{3} \mathrm{Cl}\right)+\mathrm{SnCl}_{4} \cdot 5 \mathrm{H}_{2} \mathrm{O} \rightarrow\left(n-\mathrm{C}_{8} \mathrm{H}_{17} \mathrm{NH}_{3}\right)_{2}-$ $\mathrm{SnCl}_{6}+5 \mathrm{H}_{2} \mathrm{O}$. After filtering and two recrystalizations, white sugar-like crystals were finally obtained and then 
vacuum-dried and kept in dry condition for further works. The stoichiometry and the structure were checked by elemental analysis and $\mathrm{x}$-ray diffraction (XRD). Differential scanning calorimetry (DSC) carried out between $123 \mathrm{~K}$ and $453 \mathrm{~K}$ shows three successive phase transitions in agreement with previous works $[5,6]$. The linewidth and spin-lattice relaxation time measurements were made using $200-\mathrm{MHz}{ }^{1} \mathrm{H}$ NMR in the temperature range $200-$ $380 \mathrm{~K}$.

\section{Results and Discussion}

Figure 1 shows the ${ }^{1} \mathrm{H}$ NMR FWHM (full width at half maximum) linewidth of $\mathrm{C} 8 \mathrm{Sn}$ as a function of temperature. According to previous works [5, 6] and our DSC measurements, C8Sn undergoes three successive phase transitions, at $272 \mathrm{~K}\left(T_{c 1}\right), 344 \mathrm{~K}\left(T_{c 2}\right)$, and $355 \mathrm{~K}\left(T_{c 3}\right)$, respectively. However, the linewidth reflects only two of the transitions, at around $T_{c 1}$ and $T_{c 2}$. In compounds of the $\mathrm{CnSn}$ type, a reduction of the linewidth is mainly caused by the diminution of the dipolar splitting [13]. Thus, the linear decrease of the linewidth, approaching $T_{c 1}$, can be assigned to the decrease of the dipolar splitting. Since the linewidth around $T_{c 1}$ remains fairly large, a uniaxial reorientation of the rigid alkyl chains, conserving the average chain axis, appears to be responsible for the decrease of the linewidth. Then, the transition at $T_{c \mid}$ may be ascribed to the order-disorder transition of the rigid alkyl chain.

Further decrease of the linewidth from $T_{c 1}$ to $T_{c 2}$ may arise from the disorder motion of the chain axis, which

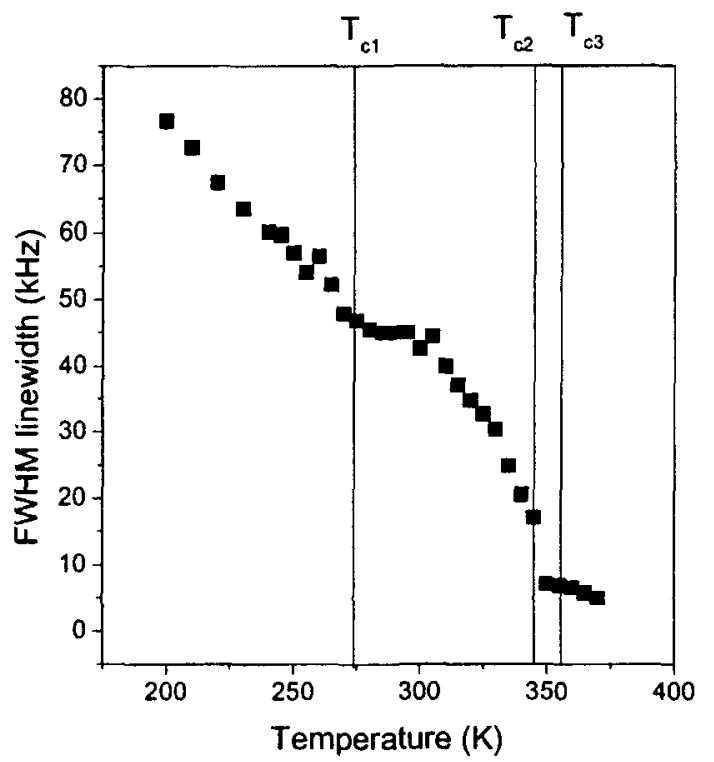

Fig. 1. 'H NMR FWHM linewidth as a function of temperature. can reduce the linewidth nearly to zero when the average chain axis is destroyed due to the activated chain defects such as the GG conformer. Then, the transition at $344 \mathrm{~K}$ is expected to be accompanied by the conformational transformation. After the average chain axis is destroyed, the hydrocarbon chains are virtually liquid-like in terms of the hydrocarbon chain motion. Since there is no average chain axis and thus no orientational order of the hydrocarbon chain, the chain appears isotropic as in a liquid and the linewidth should go below $1 \mathrm{kHz}$, as in CloSn. Around $T_{c 2}$, however, the linewidth in C8Sn remains to be about $5 \mathrm{kHz}$ and continues to decrease through $T_{c 3}$. Thus, the conformational change is believed to take place through a wide temperature range.

The spin-lattice relaxation of $\mathrm{C} 8 \mathrm{Sn}$ showed a singleexponential pattern over the whole temperature range except around $T_{c 3}$. The spin-lattice relaxation rate measurement in Fig. 2 shows a discontinuity at $T_{c 3}$, characteristic of a first-order phase transition. While $T_{c 1}$ is assigned to represent the order-disorder transition temperature in this system, no anomaly is apparent in Fig. 2 as in the case of C10Sn and C12Sn [10, 13]. In these compounds, a discontinuity in the spin-lattice relaxation time usually accompany the conformational transition. Thus, the discontinuity around $T_{c 3}$ is believed to be due to a partial chain melting originating from the conformational transformation. As discussed in Fig. 1, the conformational transition appears to take place through a wide temper-

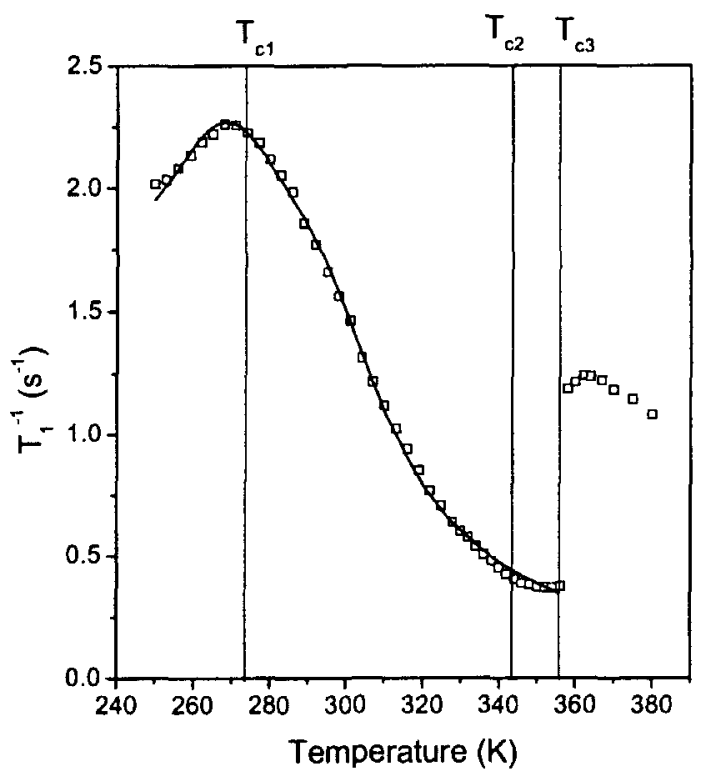

Fig. 2. The ${ }^{l} \mathrm{H}$ NMR spin-lattice relaxation rate vs temperature taken at $200 \mathrm{MHz}$. The solid line is fitted to the intramolecular dipole-dipole spin-lattice relaxation by four types of molecular motions according to Eqs. (1) and (2). 
ature range, and the partial chain melting appears to take slace at $T_{c 3}$.

The spin-lattice relaxation rate data below $T_{c 3}$ were well fitted to the intramolecular dipole-dipole interactions modulated by various types of molecular motions [14];

$$
\begin{aligned}
& T_{1}^{-1}=\Sigma_{i} \frac{2}{3} \gamma^{2} M_{2 i}\left[\frac{\tau_{c i}}{1+\left(\omega \tau_{c i}\right)^{2}}+\frac{4 \tau_{c i}}{1+\left(2 \omega \tau_{c i}\right)^{2}}\right] \\
& \quad i=1,2, \ldots, n . \\
& \tau_{c}=\tau_{0} e^{E / R T},
\end{aligned}
$$

where $\gamma$ is the proton gyromagnetic ratio, $M_{2}$ the second noment, $\omega$ the Larmor frequency and $E$ is the activation energy. Four different types of the molecular motions $n=4)$ as in the case of C10Sn were introduced to fit to he experimental data and the results of the fitting xccording to Eqs. (1) and (2) are shown in Fig. 2 as a solid line. Since the molecular motions in C8Sn are expected to be similar to those in CloSn, the second noments of the $\mathrm{CH}_{3}$ and $\mathrm{NH}_{3}$ groups acquired from ¿10Sn [10], were assumed for C8Sn.

From analysis of the spin-lattice relaxation data, the activation energies of the $\mathrm{CH}_{3}$ group were found 8,10 , and $11 \mathrm{~kJ} / \mathrm{mol}$ in C8Sn, C10Sn, and C12Sn, respectively, which values are very similar. On the other hand, the activation energies of the $\mathrm{NH}_{3}$ group are $14( \pm 4), 40$, and $.55 \mathrm{~kJ} / \mathrm{mol}$ in $\mathrm{C} 8 \mathrm{Sn}, \mathrm{C} 10 \mathrm{Sn}$, and $\mathrm{C} 12 \mathrm{Sn}$, respectively. 3elow $T_{c 1}$, an "unknown defect" motion dominates the spin-lattice relaxation as in $\mathrm{C} 10 \mathrm{Sn}$, where the second moment of the defect is about $1.7 \mathrm{G}^{2}$ and the activation inergy is about $46 \mathrm{~kJ} / \mathrm{mol}$. Near $T_{c 3}$, another type of lefect motion, presumably a chain-end defect, is dominant, 'whose second moment and activation energy are about $1.2 \mathrm{G}^{2}$ and $51 \mathrm{~kJ} / \mathrm{mol}$, respectively.

As discussed in our previous work [13], the activation snergy of the ammonium group strongly depends on the shain length and appears to be related to the orderdisorder transition of the hydrocarbon chain in the $\mathrm{CnSn}$ :ystems. The strong chain length dependence of the $\mathrm{NH}_{3}$ group is confirmed also in C8Sn. However, the presumably order-disorder transition temperatures are very close in C8Sn and C10Sn, in spite of the large difference between the activation energies of the $\mathrm{NH}_{3}$ groups. Then, the transition in C8Sn at $T_{\mathrm{c} 1}$ may not be simply assigned to an order-disorder transition.

Figure 3 shows the derivative of the spin-lattice relaxation time with respect to temperature. It shows an c.nomaly around $T_{c_{2}}$, where the conformational transition is believed to start, but the spin-lattice relaxation time itself does not show it. In CnSn systems with $n \geq 10$, the differential anomalies were also observed, around the

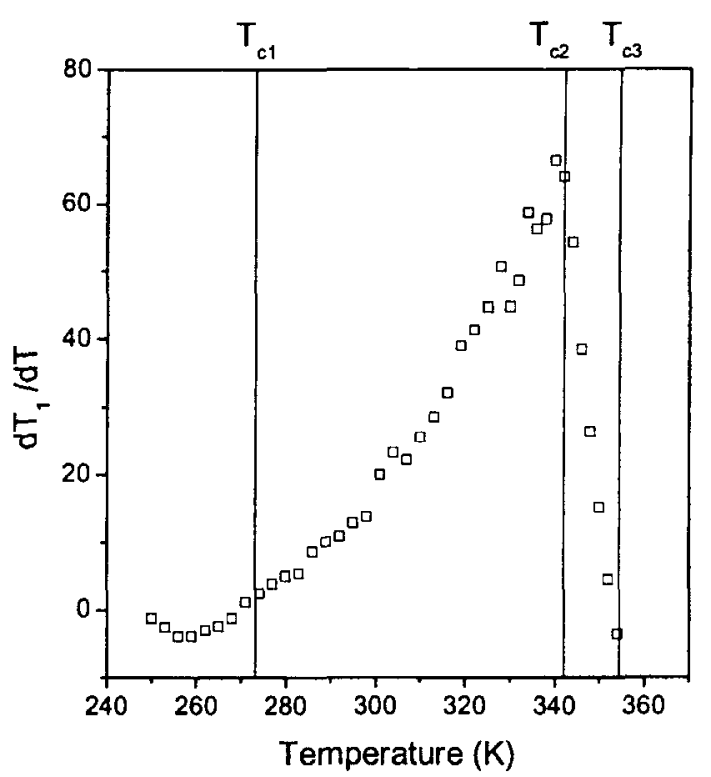

Fig. 3. Differential of the spin-lattice relaxation time with respect to temperature.

order-disorder transition temperature, which is not reflected in the spin-lattice relaxation time. In analogy to ClOSn and $\mathrm{C} 12 \mathrm{Sn}$, it can then be assumed that the transition at $T_{c 2}$ bears an order-disorder nature as well as a conformational nature. It then follows that the characteristics of the transitions in C8Sn is fairly distinct from those in C10Sn and C12Sn. In other words, both the orderdisorder and conformational natures are mixed in the phase transitions of C8Sn.

In summary, three phase transitions in $\left.\mathrm{C}_{8} \mathrm{H}_{17} \mathrm{NH}_{3}\right)_{2} \mathrm{SnCl}_{6}$ (C8Sn) were found and characterized by means of ${ }^{1} \mathrm{H}$ NMR. As a result, it was found that unlike in the $\mathrm{CnSn}$ compounds with longer hydrocarbon chains, the phase transitions in the C8Sn system have mixed nature of the hydrocarbon chain molecular motions, order-disorder and conformational dynamics.

\section{Acknowledgments}

This work was supported by the KISTEP (National Research Laboratory and Proton Accelerator User Program No. M102KS010001-02K1901-01814) and by the Brain Korea 21 Project in 2003. Measurements at the Korea Basic Science Institute (KBSI) are acknowledged.

\section{References}

[1] D. H. Brown, K. R. Dixon, C. M. Livingston, R. H. Nuttall, and O. W. A. Share, J. Chem. Soc. A 100 (1967).

[2] O. K. Knop and W. J. Westerhaus, Can. J. Chem. 58, 270 
(1980).

[3] K. Kitahama, H. Kiriyama, and Y. Baba, Bull. Chem. Soc. Jpn. 52, 324 (1979).

[4] M. H. B. Ghozlen, A. Daoud, T. Molk, H. Poulet, M. Le Postllec, and N. Toupry, J. Raman Spec. 16, 219 (1985).

[5] J. Kroupa, A. Fuith, K. J. Shenk, H. Warhanek, and M. Ceramak, Ferroelectrics 159, 109 (1994).

[6] M. Ceramak, F. Fuith, P. Vanek, J. Silha, and J. Malkova, Phys. Stat. Sol. (b) 182, 289 (1994).

[7] R. Blinc, M. I. Brugar, V. Rutar, B. Zeks, R. Kind, H. Arend, and G. Chapuis, Phys. Rev. Lett. 43, 1679 (1979).

[8] R. Kind, S. Plesko, H. Arend, R. Blinc, B. Zeks, J. Seliger, B. Lozar, J. Slak, A. Levstic, C. Filipic, V. Zagar, G. Lahajnar, F. Milia, and G. Chapuis, J. Chem. Phys. 71, 2118 (1979).
[9] C. H. Lee, K. W. Lee, C. E. Lee, and J. K. Kang, J. Korean Phys. Soc. 34, L484 (1999).

[10] K. W. Lee, M. W. Park, C. Rhee, C. E. Lee, J. K. Kang, K. W. Kim, and K. S. Lee, J. Chem. Phys. 108, 3019 (1998).

[11] K. W. Lee, C. H. Lee, C. E. Lee, and J. K. Kang, Phys. Rev. B 54, 8989 (1996).

[12] K. W. Lee, C. H. Lee, C. E. Lee, and J. K. Kang, J. Chem. Phys. 104, 6964 (1996).

[13] K. W. Lee, C. E. Lee, J. Kim, and J. K. Kang, Solid State Commun. 124, 185 (2002).

[14] C. P. Slichter, Principles of Mganetic Resonance, Spiringer-Verlag, Berlin, 1990; A. Abragam, Principles of Nuclear Magnetism, Oxford University Press, 1983. 